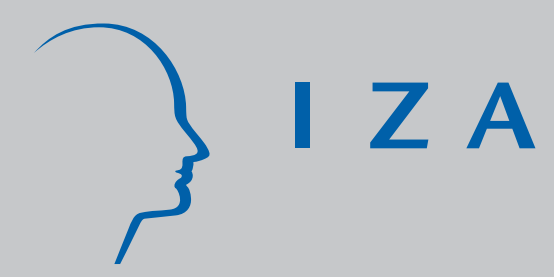

IZA DP No. 3794

An Afriat Theorem for the Collective Model of Household Consumption

Laurens Cherchye

Bram De Rock

Frederic Vermeulen

October 2008 


\title{
An Afriat Theorem for the Collective Model of Household Consumption
}

\author{
Laurens Cherchye \\ University of Leuven \\ and FWO-Vlaanderen \\ Bram De Rock \\ ECARES and ECORE, \\ Université Libre de Bruxelles \\ Frederic Vermeulen \\ CentER, Tilburg University, \\ Netspar and IZA
}

\section{Discussion Paper No. 3794 \\ October 2008}

\author{
IZA \\ P.O. Box 7240 \\ 53072 Bonn \\ Germany \\ Phone: +49-228-3894-0 \\ Fax: +49-228-3894-180 \\ E-mail: iza@iza.org
}

\begin{abstract}
Any opinions expressed here are those of the author(s) and not those of IZA. Research published in this series may include views on policy, but the institute itself takes no institutional policy positions.

The Institute for the Study of Labor (IZA) in Bonn is a local and virtual international research center and a place of communication between science, politics and business. IZA is an independent nonprofit organization supported by Deutsche Post World Net. The center is associated with the University of Bonn and offers a stimulating research environment through its international network, workshops and conferences, data service, project support, research visits and doctoral program. IZA engages in (i) original and internationally competitive research in all fields of labor economics, (ii) development of policy concepts, and (iii) dissemination of research results and concepts to the interested public.
\end{abstract}

IZA Discussion Papers often represent preliminary work and are circulated to encourage discussion. Citation of such a paper should account for its provisional character. A revised version may be available directly from the author. 
IZA Discussion Paper No. 3794

October 2008

\section{ABSTRACT}

\section{An Afriat Theorem for the Collective Model of Household Consumption ${ }^{*}$}

We provide a nonparametric 'revealed preference' characterization of rational household behavior in terms of the collective consumption model, while accounting for general (possibly non-convex) individual preferences. We establish a Collective Axiom of Revealed Preference $(C A R P)$, which provides a necessary and sufficient condition for data consistency with collective rationality. Our main result takes the form of a 'collective' version of the Afriat Theorem for rational behavior in terms of the unitary model. This theorem has some interesting implications. With only a finite set of observations, the nature of consumption externalities (positive or negative) in the intra-household allocation process is non-testable. The same non-testability conclusion holds for privateness (with or without externalities) or publicness of consumption. By contrast, concavity of individual utility functions (representing convex preferences) turns out to be testable. In addition, monotonicity is testable for the model that assumes all household consumption is public.

JEL Classification: D11, D12, D13, C14

Keywords: collective model, consumption, Pareto efficiency, revealed preferences, Afriat theorem, Collective Axiom of Revealed Preferences

Corresponding author:

Frederic Vermeulen

Tilburg University

P.O. Box 90153

NL-5000 LE Tilburg

The Netherlands

E-mail: frederic.vermeulen@uvt.nl

* Frederic Vermeulen gratefully acknowledges financial support from the Netherlands Organisation for Scientific Research (NWO) through a VIDI grant. 


\section{Introduction}

The collective model has become increasingly popular to analyze household consumption behavior. Chiappori $(1988,1992)$ introduced this model as a valuable alternative for the standard unitary model, which describes household behavior as if the household were a single decision maker, who maximizes 'household' preferences subject to the household budget constraint. The collective model explicitly recognizes that the household consists of multiple decision makers (household members) with own rational preferences. It only assumes that the observed household consumption decisions are Pareto efficient outcomes of an intra-household allocation process.

Browning and Chiappori (1998) suggested a most general collective consumption model, which accounts for externalities and public consumption within the household. In addition, they make the minimalistic assumption that the empirical analyst does not observe which consumption quantities are privately consumed (possibly characterized by externalities) and which quantities are publicly consumed. Focusing on a parametric characterization of this general model, they establish that for two-person households collectively rational household behavior requires a pseudo-Slutsky matrix that can be written as the sum of a symmetric negative semi-definite matrix and a rank one matrix. Browning and Chiappori show necessity of this condition; Chiappori and Ekeland (2006) address the associated sufficiency question.

For this general collective consumption model, Cherchye, De Rock and Vermeulen (2007) recently presented a nonparametric 'revealed preference' characterization in the tradition of Afriat (1967) and Varian (1982). ${ }^{1}$ They established a necessary nonparametric condition and a complementary sufficient nonparametric condition that allow for testing whether a finite number of observations can be rationalized in terms of the collective model. They argue that, in general, the necessary condition and the sufficient condition do not coincide. For deriving their results, these authors assume concave utility functions (representing convex preferences) of the individual household members; this implies a convex utility possibility set and a corresponding characterization of Pareto efficient intra-household allocations. In addition, they exclude negative externalities. Note that a similar convexity assumption is made by Browning and Chiappori (1998) and Chiappori and Ekeland

\footnotetext{
${ }^{1}$ See also Samuelson (1938), Houthakker (1950) and Diewert (1973) for seminal contributions on the revealed preference approach to analyzing consumption behavior.
} 
(2006) in their parametric setting.

We complete the results of Cherchye, De Rock and Vermeulen (2007). More specifically, we address the same questions but we drop the prior assumptions that members' preferences are convex and that there are no negative externalities. Relaxing convexity implies that the household utility possibility set can be non-convex (even if the budget set is linear). Indeed, it has been argued that convexity assumptions are problematic in the presence of (positive or negative) externalities; see for example Starr (1969), Starret (1972) and, more recently, Mas-Colell, Whinston and Green (1995). In addition, the fact that we do not impose convexity a priori is consistent with the nonparametric 'revealed preference' approach for analyzing the unitary model, which equally does not assume convex (in casu unitary 'household') preferences a priori but only maintains local non-satiation as a minimal assumption (see, e.g., Varian, 1982). In our approach, we only maintain a local collective non-satiation assumption which, as we will argue, provides a natural 'collective' version of the non-satiation assumption that is used in the context of the unitary model.

Our main result is that we derive a nonparametric 'revealed preference' condition in terms of the observed aggregate quantity and price data that is both necessary and sufficient for household behavior to be consistent with Pareto efficiency under general preferences of the household members. Specifically, we show that the condition which Cherchye, De Rock and Vermeulen (2007) identified as necessary (but not sufficient) for a collective rationalization of the data under convex individual preferences, becomes both necessary and sufficient when dropping the convex preferences assumption. The condition has a formally similar structure as the Generalized Axiom of Revealed Preference $(G A R P)$ that provides a necessary and sufficient condition for consistency of observed household behavior with the unitary model (see Varian, 1982); and, therefore, we call it the Collective Axiom of Revealed Preference $(C A R P)$. This characterization of collectively rational behavior in terms of data consistency with $C A R P$ takes the form of a 'collective' version of the well-known Afriat Theorem for the unitary model.

Apart from the general collective consumption model, we also consider two special cases of this general model: (i) the model that excludes public consumption and externalities (also known as the 'egoistic' model); and (ii) the model that excludes private consumption (and, thus, in which all con- 
sumption is public). ${ }^{2}$ Chiappori and Ekeland (2006) use the same distinction between a general case and special cases to structure their parametric results on empirical characterizations of collective consumption models. Moreover, the special cases are mostly considered in empirical applications of the collective model. Interestingly, we find that data consistency with $C A R P$ is also necessary and sufficient for a data rationalization in terms of these special cases. This implies that assumptions regarding privateness or publicness of consumption are non-testable under the abovementioned assumptions. In fact, as we will discuss, our results yield some additional testability conclusions regarding properties (concavity and monotonicity) of the data rationalizing utility functions. We will contrast these results with existing findings on the (non)parametric characterization of collective consumption models under convex preferences, and on the nonparametric characterization of the unitary model.

From a practical point of view, because $C A R P$ only includes observable price and quantity information, our results directly imply a necessary and sufficient 'revealed preference' condition for collective rationality that can be tested on a finite number of observations. Because of our minimal prior assumptions, this $C A R P$ test can be conceived as a 'pure test' of Pareto efficient collective consumption behavior. Interestingly, this test applies to a general number of observations and has direct practical applicability. It follows from Proposition 3 of Cherchye, De Rock and Vermeulen (2007) that collectively rational behavior (summarized in terms of $C A R P$ ) can be rejected if and only if there are at least three observations and three goods. Cherchye, De Rock, Sabbe and Vermeulen (2008) present an integer programming version of the test, including a MATLAB code and an empirical application to real-life data.

At this point, it is worth indicating that our results can also be instrumental in alternative contexts. For example, they readily extend to the general case of multi-person group consumption. See Chiappori and Ekeland

\footnotetext{
${ }^{2}$ The 'egoistic' model actually encompasses a wider class of member-specific utilities, which model altruism in a specific way: it also includes so-called caring preferences of the individual household members, which depend not only on the member's own (egoistic) utility but also on the other member's utility. Chiappori (1992) argues that, given Pareto efficiency, the empirical implications of caring preferences are indistinguishable from those of egoistic preferences. As such, while we will not indicate this explicitly in the following discussion, our conclusions for the egoistic model carry over to the (more general) caring model.
} 
(2006, 2008) for discussion on the relevance of the collective model within the context of group consumption. To ease our exposition, the theoretical discussion in the following sections focuses on two-person households. Generalizations for $M$-member groups $(M \geq 2)$ can be obtained along the lines of Cherchye, De Rock and Vermeulen (2007; supplemental material). Next, the nonparametric approach to analyzing collective consumption behavior is closely related to the literature on testable nonparametric restrictions of general equilibrium models. See Brown and Matzkin (1996), Brown and Shannon (2000) and, for a more recent survey, Carvajal, Ray and Snyder (2004). As such, our insights can be useful to conceive nonparametric general equilibrium restrictions in the case of non-convex preferences. Lastly, our results for the collective consumption model can also be relevant for nonparametric production analysis. See Cherchye De Rock and Vermeulen (2008), who adopt a formally similar collective model for analyzing economies of scope in the context of multi-output production (in casu under convex output possibility sets).

Before entering our analysis, two final remarks are in order. First, parametric applications of the collective model often use so-called 'assignable' quantity information, which means that the empirical analyst observes how much a group member consumes of the corresponding goods; see, for example, Browning, Bourguignon, Chiappori and Lechene (1994) and Bourguignon, Browning and Chiappori (2008). Such information is often partly, but not fully, available in practical applications (through budget surveys). To keep our discussion focused, we will abstract from such assignable quantity information in what follows. Still, it is worth emphasizing that including such information in our following results is relatively easy; it can proceed analogously as in Cherchye, De Rock and Vermeulen (2008), who focus on nonparametric testing and recovery (or 'identifiability') of collective consumption models under the maintained assumption of convex preferences, while accounting for assignable quantity information.

The second introductory remark pertains to the precise interpretation of our following testability conclusions regarding concave utility functions; see in particular our discussion in Section 6. In this respect, it is worth to briefly recall the subtle relation between 'convex preferences' and 'concave utility functions'. Concave utility functions always imply a convex utility possibility set (when the budget set is linear). In other words, if we reject collective rationality for a convex utility possibility set, then we also reject collective rationality for concave utility functions. Thus, testability of a convex 
utility possibility set relates unambiguously to testability of concave utility functions. But it is also well-known that, under some mild technical conditions, convex preferences can be represented by a concave utility function; see Kannai (1977) and Richter and Wong (2004) for discussion. As such, when assuming these mild conditions, our testability results regarding concave utility functions directly extend to convex preferences. Given this, we will sometimes refer to convex preferences instead of concave utility functions in the following.

The remainder of this study is structured as follows. Section 2 sets the stage by briefly recapturing the unitary GARP condition and the corresponding Afriat Theorem. Section 3 defines collective rationality. Section 4 provides the associated nonparametric CARP condition and the Collective Afriat Theorem. Section 5 focuses on two special cases of the collective model. Section 6 relates our findings to existing results in the literature. Finally, Section 7 concludes. The Appendix contains the proofs of our main results.

\section{Unitary rationality}

We first recapture the nonparametric conditions for unitary rationality, as they have been presented by Varian (1982). This will ease our following discussion, as it enables comparing our main results for the collective rationality model with those for the unitary rationality model.

We consider a household that purchases the (non-zero) $N$-vector of quantities $\mathbf{q} \in \mathbb{R}_{+}^{N}$ when confronted with the prices $\mathbf{p} \in \mathbb{R}_{++}^{N}$. Suppose $T$ observations of the household consumption behavior. For each observation $t$, we use $\mathbf{p}_{t}$ and $\mathbf{q}_{t}$ to denote the (observed) aggregate prices and quantities, respectively; while $S=\left\{\left(\mathbf{p}_{t} ; \mathbf{q}_{t}\right), t=1, \ldots, T\right\}$ represents the set of observations. We can then define the condition for a unitary rationalization of a set of observations $S$, which -to recall- models household behavior as if the household were a single decision maker (i.e. the household maximizes a single utility function).

Definition 1 (unitary rationalization) Let $S=\left\{\left(\mathbf{p}_{t} ; \mathbf{q}_{t}\right) ; t=1, \ldots, T\right\}$ be a set of observations. A utility function $U$ provides a unitary rationalization of $S$ if for each observation $t$ we have $U\left(\mathbf{q}_{t}\right) \geq U\left(\mathbf{q}_{r}\right)$ for all $\mathbf{q}_{r}$ with $\mathbf{p}_{t}^{\prime} \mathbf{q}_{r} \leq$ $\mathbf{p}_{t}^{\prime} \mathbf{q}_{t}$. 
The nonparametric condition for unitary rationality only assumes local non-satiation of the utility function $U$. As argued by Varian (1982), local non-satiation avoids trivial rationalizations of the data for the unitary model: without this additional assumption, any observed household consumption behavior can be rationalized in terms of this model.

Definition 2 (local non-satiation) A utility function $U$ satisfies local nonsatiation if the following holds. Suppose quantities $\mathbf{q}_{r}$. Then for any $\epsilon>0$ there exist quantities $\mathbf{q}$ with $\left\|\mathbf{q}-\mathbf{q}_{r}\right\|<\epsilon$ such that $U(\mathbf{q})>U\left(\mathbf{q}_{r}\right)$.

Varian (1982) established that there exists a locally non-satiated utility function that provides a unitary rationalization of the set of observations $S$ if and only if the data satisfy the Generalized Axiom of Revealed Preference $(G A R P)$.

Definition 3 (GARP) Let $S=\left\{\left(\mathbf{p}_{t} ; \mathbf{q}_{t}\right) ; t=1, \ldots, T\right\}$ be a set of observations. The set $S$ satisfies the Generalized Axiom of Revealed Preference (GARP) if there exist relations $R_{0}, R$ that meet:

(i) if $\mathbf{p}_{s}^{\prime} \mathbf{q}_{s} \geq \mathbf{p}_{s}^{\prime} \mathbf{q}_{t}$ then $\mathbf{q}_{s} R_{0} \mathbf{q}_{t}$;

(ii) if $\mathbf{q}_{s} R_{0} \mathbf{q}_{u}, \mathbf{q}_{u} R_{0} \mathbf{q}_{v}, \ldots, \mathbf{q}_{z} R_{0} \mathbf{q}_{t}$ for some (possibly empty) sequence $(u, v, \ldots, z)$ then $\mathbf{q}_{s} R \mathbf{q}_{t}$;

(iii) if $\mathbf{q}_{s} R \mathbf{q}_{t}$ then $\mathbf{p}_{t}^{\prime} \mathbf{q}_{t} \leq \mathbf{p}_{t}^{\prime} \mathbf{q}_{s}$.

In words, the quantities $\mathbf{q}_{s}$ are 'directly revealed preferred' over the quantities $\mathbf{q}_{t}$ (i.e. $\mathbf{q}_{s} R_{0} \mathbf{q}_{t}$ ) if $\mathbf{q}_{s}$ were chosen when $\mathbf{q}_{t}$ were equally attainable (i.e. $\mathbf{p}_{s}^{\prime} \mathbf{q}_{s} \geq \mathbf{p}_{s}^{\prime} \mathbf{q}_{t}$ ); see rule (i). Next, the 'revealed preference' relation $R$ exploits transitivity of preferences; see rule (ii). Finally, rule (iii) imposes that the quantities $\mathbf{q}_{t}$ cannot be more expensive than revealed preferred quantities $\mathbf{q}_{s}$.

The following Afriat Theorem (Varian, 1982; based on Afriat, 1967) gives a nonparametric characterization of rational consumption behavior in terms of the unitary model.

Theorem 1 (Afriat Theorem) Let $S=\left\{\left(\mathbf{p}_{t} ; \mathbf{q}_{t}\right) ; t=1, \ldots, T\right\}$ be a set of observations. The following statements are equivalent:

(i) There exists a utility function $U$ that satisfies local non-satiation and that provides a unitary rationalization of $S$;

(ii) The set $S$ satisfies GARP; 
(iii) For all $t, r \in\{1, \ldots, T\}$, there exist numbers $U_{t}, \lambda_{t} \in \mathbb{R}_{++}$that meet the Afriat inequalities

$$
U_{r}-U_{t} \leq \lambda_{t} \mathbf{p}_{t}^{\prime}\left(\mathbf{q}_{r}-\mathbf{q}_{t}\right)
$$

(iv) There exists a continuous, monotonically increasing and concave utility function $U$ that satisfies local non-satiation and that provides a unitary rationalization of $S$.

In this result, condition (ii) implies that data consistency with GARP is necessary and sufficient for a unitary rationalization of the data. Condition (iii) provides an equivalent characterization in terms of the Afriat inequalities, which allow an explicit construction of the utility levels associated with each observation $t$ (i.e. utility level $U_{t}$ for observed quantities $\mathbf{q}_{t}$ ). Finally, condition (iv) states that, if there exists a utility function that provides a unitary rationalization of the set $S$, then there exists a continuous, monotone and concave utility function that provides such a rationalization. This also implies that continuity, monotonicity and concavity of the data rationalizing utility function is non-testable for the unitary model; i.e., violations of continuity, monotonicity or concavity cannot be detected with a finite number of observations.

\section{Collective rationality}

We consider a two-member (1 and 2) household. Like before, the household purchases the (non-zero) $N$-vector of quantities $\mathbf{q} \in \mathbb{R}_{+}^{N}$ with corresponding prices $\mathbf{p} \in \mathbb{R}_{++}^{N}$. All goods can be consumed privately, publicly or both. Generally, we have $\mathbf{q}=\mathbf{q}^{1}+\mathbf{q}^{2}+\mathbf{q}^{h}$ for $\mathbf{q}$ the (observed) aggregate quantities, $\mathbf{q}^{1}$ and $\mathbf{q}^{2}$ the (unobserved) private quantities of each household member, and $\mathbf{q}^{h}$ the (unobserved) public quantities.

Following Browning and Chiappori (1998), we consider general preferences for the household members that may depend not only on the own private and public quantities, but also on the other individual's private quantities; this allows for (positive or negative) externalities. Formally, this means that the preferences of each household member $m(m=1,2)$ can be represented by a utility function of the form $U^{m}$ that is defined in the arguments $\mathbf{q}^{1}, \mathbf{q}^{2}$ and $\mathbf{q}^{h}$.

For aggregate quantities $\mathbf{q}$, we define feasible personalized quantities $\widehat{\mathbf{q}}$ as

$$
\widehat{\mathbf{q}}=\left(\mathfrak{q}^{1}, \mathfrak{q}^{2}, \mathfrak{q}^{h}\right) \text { with } \mathfrak{q}^{1}, \mathfrak{q}^{2}, \mathfrak{q}^{h} \in \mathbb{R}_{+}^{n} \text { and } \mathfrak{q}^{1}+\mathfrak{q}^{2}+\mathfrak{q}^{h}=\mathbf{q}
$$


Each $\widehat{\mathbf{q}}$ captures a feasible decomposition of the aggregate quantities $\mathbf{q}$ into private quantities $\left(\mathfrak{q}^{1}\right.$ and $\left.\mathfrak{q}^{2}\right)$ and public quantities $\left(\mathfrak{q}^{h}\right)$. This reflects that the general model allows for both private and public consumption; for the two special cases mentioned in the introduction, some of these components of $\widehat{\mathbf{q}}$ are zero by construction (see also Section 5). In the following, we consider feasible personalized quantities because we assume that the 'true' personalized quantities are not observed. Throughout, we will use that each $\widehat{\mathbf{q}}$ defines a unique $\mathbf{q}$.

Given this, a collective rationalization of $S$ requires the existence of utility functions $U^{1}$ and $U^{2}$ such that each observed consumption bundle can be characterized as Pareto efficient.

Definition 4 (collective rationalization) Let $S=\left\{\left(\mathbf{p}_{t} ; \mathbf{q}_{t}\right) ; t=1, \ldots, T\right\}$ be a set of observations. A pair of utility functions $U^{1}$ and $U^{2}$ provides a collective rationalization of $S$ if for each observation there exist feasible personalized quantities $\widehat{\mathbf{q}}_{t}$ such that $U^{m}\left(\widehat{\mathbf{q}}_{r}\right)>U^{m}\left(\widehat{\mathbf{q}}_{t}\right)$ implies $U^{l}\left(\widehat{\mathbf{q}}_{r}\right)<$ $U^{l}\left(\widehat{\mathbf{q}}_{t}\right)(m \neq l)$ for all feasible personalized quantities $\widehat{\mathbf{q}}_{r}$ with $\mathbf{p}_{t}^{\prime} \mathbf{q}_{r} \leq \mathbf{p}_{t}^{\prime} \mathbf{q}_{t}$.

Just like the nonparametric condition for unitary rationality assumes local non-satiation, we will assume local collective non-satiation of the individual utility functions $U^{1}$ and $U^{2}$. Because it has a formally similar structure, this local collective non-satiation concept can be interpreted as the 'collective' analogue of the local non-satiation concept in Definition 2. In what follows, we will only use local collective non-satiation as a maintained assumption. As such, and in contrast with Cherchye, De Rock and Vermeulen (2007), we do not maintain concavity and (positive) monotonicity of the individual utility functions. Inter alia, this implies that we do not a priori exclude negative consumption externalities.

Definition 5 (local collective non-satiation) A pair of utility functions $U^{1}$ and $U^{2}$ satisfies local collective non-satiation if the following holds. Suppose feasible personalized quantities $\widehat{\mathbf{q}}_{r_{1}}$ and $\widehat{\mathbf{q}}_{r_{2}}$, and let $\mathbf{r}=\left\{r_{1}\right\} \cup\left\{r_{2}\right\}$. Then for any $\epsilon \geq 0$ there exist quantities $\mathbf{q}$ with $\left\|\sum_{r \in \mathbf{r}} \mathbf{q}_{r}-\mathbf{q}\right\| \leq \epsilon$ such that $U^{1}(\widehat{\mathbf{q}}) \geq U^{1}\left(\widehat{\mathbf{q}}_{r_{1}}\right)$ and $U^{2}(\widehat{\mathbf{q}}) \geq U^{2}\left(\widehat{\mathbf{q}}_{r_{2}}\right)$, with at least one strict inequality if $\epsilon>0$, for feasible personalized quantities $\widehat{\mathbf{q}}$.

In words, suppose an initial situation in which member 1 evaluates feasible personalized quantities $\widehat{\mathbf{q}}_{r_{1}}$ (corresponding to $\mathbf{q}_{r_{1}}$ ) and member 2 evaluates 
feasible personalized quantities $\widehat{\mathbf{q}}_{r_{2}}$ (corresponding to $\mathbf{q}_{r_{2}}$ ). Then for $\epsilon>$ 0 and $\mathbf{q}$ close to the 'summed quantities' $\sum_{r \in \mathbf{r}} \mathbf{q}_{r}$ there exist personalized quantities $\widehat{\mathbf{q}}$ that entail a Pareto improvement as compared to this initial situation. ${ }^{3}$ As for the specification of $\sum_{r \in \mathbf{r}} \mathbf{q}_{r}$, we need to consider two cases. In the first case, $r_{1}=r_{2}$ and thus $\sum_{r \in \mathbf{r}} \mathbf{q}_{r}=\mathbf{q}_{r_{1}}=\mathbf{q}_{r_{2}}$. This pertains to an initial situation in which both household members evaluate the same feasible personalized quantities $\widehat{\mathbf{q}}_{r_{1}}=\widehat{\mathbf{q}}_{r_{2}}$; and local collective non-satiation implies that a Pareto improvement is possible for (aggregate) quantities $\mathbf{q}$ close to $\mathbf{q}_{r_{1}}=\mathbf{q}_{r_{2}}$. In the second case, $r_{2} \neq r_{1}$ and thus $\sum_{r \in \mathbf{r}} \mathbf{q}_{r}=\mathbf{q}_{r_{1}}+\mathbf{q}_{r_{2}}$. This pertains to an initial situation in which both household members evaluate different feasible personalized quantities $\widehat{\mathbf{q}}_{r_{1}}$ and $\widehat{\mathbf{q}}_{r_{2}}$; and local collective non-satiation implies that a Pareto improvement is possible for (aggregate) quantities $\mathbf{q}$ close to the sum $\mathbf{q}_{r_{1}}+\mathbf{q}_{r_{2}}$.

This assumption of local collective non-satiation avoids trivial collective rationalizations of a set of observations $S$ : without this assumption, any observed household consumption behavior can be rationalized in terms of the collective consumption model (i.e. any set $S$ of quantity choices q under alternative prices $\mathbf{p}$ can be characterized as Pareto efficient intra-household allocations). ${ }^{4}$ As such, this maintained assumption plays exactly the same role for the collective model as the standard local non-satiation assumption for the unitary model.

To conclude this section, we introduce an equivalent characterization of Pareto efficiency in Definition 4, which will be useful for our following discussion:

Lemma 1 Let $S=\left\{\left(\mathbf{p}_{t} ; \mathbf{q}_{t}\right) ; t=1, \ldots, T\right\}$ be a set of observations. A pair of utility functions $U^{1}$ and $U^{2}$ provides a collective rationalization of $S$ if and

\footnotetext{
${ }^{3}$ As compared to Definition 2, we also consider $\epsilon=0$ in Definition 5. The condition for $\epsilon=0$ is a technical one, which is only required in a limiting case, i.e. $\mathbf{r}=\left\{t_{1}, t_{2}\right\}$ and $\mathbf{p}_{s}^{\prime} \mathbf{q}_{s}=\mathbf{p}_{s}^{\prime}\left(\mathbf{q}_{t_{1}}+\mathbf{q}_{t_{2}}\right)$, to obtain rule (iii) in Definition 6 (Collective Axiom of Revealed Preference; $C A R P$ ) as a necessary condition for a collective rationalization of a set of observations $S$. See Step 1 in the proof of Theorem 2.

${ }^{4}$ More specifically, local collective non-satiation in Definition 5 is crucial to obtain rules (iii) (for $\mathbf{r}=\left\{t_{1}, t_{2}\right\}$ with $\left.t_{1} \neq t_{2}\right)$ and (iv) in Definition $6(C A R P)$ as necessary conditions for a collective rationalization of a set of observations $S$; see Step 1 in the proof of Theorem 2 . Without these rules, any set $S$ trivially satisfies the remaining rules in the definition of $C A R P$. For example, it can be verified that, in this case, consistency of any set $S$ with $C A R P$ is achieved for a transitive specification of the relations $H^{1}, H^{2}$ which satisfies, for all $\mathbf{q}_{s}$ and $\mathbf{q}_{t}$, that $\mathbf{q}_{s} H^{1} \mathbf{q}_{t}$ implies $\mathbf{q}_{t} H^{2} \mathbf{q}_{s}$.
} 
only if for each observation $t$ there exist feasible personalized quantities $\widehat{\mathbf{q}}_{t}$ such that, for all $\widehat{\mathbf{q}}_{r}$ with $\mathbf{p}_{t}^{\prime} \mathbf{q}_{r} \leq \mathbf{p}_{t}^{\prime} \mathbf{q}_{t}$, there exist $\mu_{t r}^{1}$ and $\mu_{t r}^{2} \in \mathbb{R}_{++}$that imply

$$
\mu_{t r}^{1} U^{1}\left(\widehat{\mathbf{q}}_{t}\right)+\mu_{t r}^{2} U^{2}\left(\widehat{\mathbf{q}}_{t}\right) \geq \mu_{t r}^{1} U^{1}\left(\widehat{\mathbf{q}}_{r}\right)+\mu_{t r}^{2} U^{2}\left(\widehat{\mathbf{q}}_{r}\right)
$$

This alternative characterization of collective rationality (or Pareto efficiency) will return in the collective Afriat inequalities that we introduce below (Theorem 2). Essentially, it requires that for observation $t$ to correspond to a Pareto efficient intrahousehold allocation there must exist $\widehat{\mathbf{q}}_{t}$ such that, for any $\widehat{\mathbf{q}}_{r}$ that was equally attainable under the given prices (i.e. with $\mathbf{p}_{t}^{\prime} \mathbf{q}_{r} \leq \mathbf{p}_{t}^{\prime} \mathbf{q}_{t}$ ), we can define strictly positive weights $\mu_{t r}^{1}$ and $\mu_{t r}^{2}$ for which the corresponding weighted sum of utilities for $\widehat{\mathbf{q}}_{t}$ exceeds the one for $\widehat{\mathbf{q}}_{r}$ (i.e. $\left.\mu_{t r}^{1} U^{1}\left(\widehat{\mathbf{q}}_{t}\right)+\mu_{t r}^{2} U^{2}\left(\widehat{\mathbf{q}}_{t}\right) \geq \mu_{t r}^{1} U^{1}\left(\widehat{\mathbf{q}}_{r}\right)+\mu_{t r}^{2} U^{2}\left(\widehat{\mathbf{q}}_{r}\right)\right)$. We note that, for each $t$, the weights $\mu_{t r}^{1}$ and $\mu_{t r}^{2}$ depend on the identity of $\widehat{\mathbf{q}}_{r}$. This follows from the fact we do not assume concave utility functions for the individual members, which -to recall- implies that we may have a non-convex utility possibility set (even if the budget set is linear). If we would have assumed a convex utility possibility set (e.g. because of concave individual utility functions representing convex preferences), then we could specify $\mu_{t r}^{m}=\mu_{t}^{m}(m=1,2)$ for all $\widehat{\mathbf{q}}_{r}$; this case is usually considered in the literature on collective consumption models.

We illustrate by Figure 1, which presents a non-convex utility possibility set corresponding to some given budget. In that figure, $\widehat{\mathbf{q}}_{s}$ (corresponding to point 1 in Figure 1) is Pareto inefficient: the intersection of the utility possibility set with the light shaded area is non-empty. For example, $\widehat{\mathbf{q}}_{t}$ (point 2) implies a Pareto improvement over $\widehat{\mathbf{q}}_{s}$, because $U^{1}\left(\widehat{\mathbf{q}}_{t}\right)>U^{1}\left(\widehat{\mathbf{q}}_{s}\right)$ and $U^{2}\left(\widehat{\mathbf{q}}_{t}\right)>U^{2}\left(\widehat{\mathbf{q}}_{s}\right)$. In terms of the characterization in Lemma 1, we have $\mu_{s t}^{1} U^{1}\left(\widehat{\mathbf{q}}_{s}\right)+\mu_{s t}^{2} U^{2}\left(\widehat{\mathbf{q}}_{s}\right)<\mu_{s t}^{1} U^{1}\left(\widehat{\mathbf{q}}_{t}\right)+\mu_{s t}^{2} U^{2}\left(\widehat{\mathbf{q}}_{t}\right)$ for each possible specification of $\mu_{s t}^{1}$ and $\mu_{s t}^{2}$. By contrast, $\widehat{\mathbf{q}}_{t}$ is Pareto efficient: the intersection of the utility possibility set with the dark shaded area is empty. Correspondingly, $\widehat{\mathbf{q}}_{t}$ is consistent with the Pareto efficiency characterization in Lemma 1.

The figure also allows us to illustrate the difference between the characterization of Pareto efficiency for non-convex utility possibility sets with the one for convex utility possibility sets: for the given (non-convex) utility possibility set, $\widehat{\mathbf{q}}_{t}$ is Pareto efficient when compared to the member-specific utilities that correspond to points 3 and 4, while there exist convex combinations of these member-specific utilities that imply a Pareto improvement over $\widehat{\mathbf{q}}_{t}$. This difference pertains to the fact that in Lemma 1, for each $t$, the 


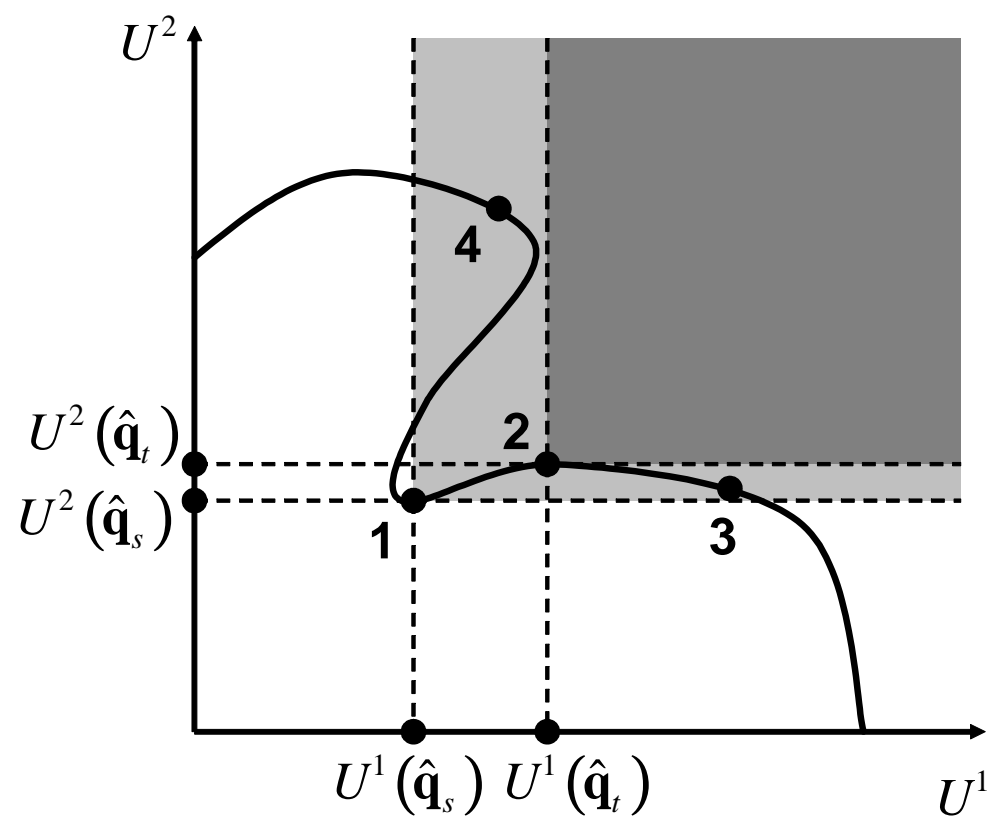

Figure 1: Pareto efficiency

Pareto weights $\mu_{t r}^{1}$ and $\mu_{t r}^{2}$ can vary with the identity of $r$.

\section{Nonparametric 'revealed preference' char- acterization}

We first present the Collective Axiom of Revealed Preference (CARP), which we can interpret as a natural 'collective' extension of the unitary GARP condition. CARP imposes empirical restrictions on hypothetical preference relations $H_{0}^{m}$ and $H^{m}$, which capture 'feasible' specifications of the individual preference relations given the information that is revealed by the set of observations $S: \mathbf{q}_{s} H^{m} \mathbf{q}_{t}\left(\mathbf{q}_{s} H_{0}^{m} \mathbf{q}_{t}\right)$ means that we 'hypothesize' that member $m$ (directly) prefers the quantities $\mathbf{q}_{s}$ over the quantities $\mathbf{q}_{t}$. Note that, while the 'true' preferences are expressed in terms of the feasible personalized quantities $\widehat{\mathbf{q}}$ (i.e. member $m$ prefers $\mathbf{q}_{s}$ over $\mathbf{q}_{t}$ only if $U^{m}\left(\widehat{\mathbf{q}}_{s}\right) \geq U^{m}\left(\widehat{\mathbf{q}}_{t}\right)$ ), the hypothetical preferences only use observable information (captured by the observed aggregate prices $\mathbf{p}$ and quantities $\mathbf{q}$ in the set $S$ ). This nat- 
urally complies with the assumption that in the general model we have no information concerning the feasible personalized quantities.

Definition 6 (CARP) Let $S=\left\{\left(\mathbf{p}_{t} ; \mathbf{q}_{t}\right) ; t=1, \ldots, T\right\}$ be a set of observations. The set $S$ satisfies the Collective Axiom of Revealed Preference (CARP) if there exist hypothetical relations $H_{0}^{m}, H^{m}$ for each member $m \in\{1,2\}$ that meet:

(i) if $\mathbf{p}_{s}^{\prime} \mathbf{q}_{s} \geq \mathbf{p}_{s}^{\prime} \mathbf{q}_{t}$ then $\mathbf{q}_{s} H_{0}^{1} \mathbf{q}_{t}$ or $\mathbf{q}_{s} H_{0}^{2} \mathbf{q}_{t}$;

(ii) if $\mathbf{q}_{s} H_{0}^{m} \mathbf{q}_{u}, \mathbf{q}_{u} H_{0}^{m} \mathbf{q}_{v}, \ldots, \mathbf{q}_{z} H_{0}^{m} \mathbf{q}_{t}$ for some (possibly empty) sequence $(u, v, \ldots, z)$ then $\mathbf{q}_{s} H^{m} \mathbf{q}_{t}$;

(iii) for $\mathbf{r}=\left\{t_{1}\right\} \cup\left\{t_{2}\right\}$ : if $\mathbf{p}_{s}^{\prime} \mathbf{q}_{s} \geq \mathbf{p}_{s}^{\prime} \sum_{r \in \mathbf{r}} \mathbf{q}_{r}$ and $\mathbf{q}_{t_{1}} H^{m} \mathbf{q}_{s}$ then $\mathbf{q}_{s} H_{0}^{l} \mathbf{q}_{t_{2}}$ (with $m \neq l$ );

(iv) if $\mathbf{q}_{s_{1}} H^{1} \mathbf{q}_{t}$ and $\mathbf{q}_{s_{2}} H^{2} \mathbf{q}_{t}$ then $\mathbf{p}_{t}^{\prime} \mathbf{q}_{t} \leq \mathbf{p}_{t}^{\prime} \sum_{r \in \mathbf{r}} \mathbf{q}_{r}$ for $\mathbf{r}=\left\{s_{1}\right\} \cup\left\{s_{2}\right\}$.

This $C A R P$ axiom has a direct interpretation in terms of the Pareto efficiency requirement that underlies collective rationality. Rule (i) states that, if the quantities $\mathbf{q}_{s}$ were chosen while the quantities $\mathbf{q}_{t}$ were equally attainable (under the prices $\mathbf{p}_{s}$ ), then it must be that at least one member prefers the quantities $\mathbf{q}_{s}$ over the quantities $\mathbf{q}_{t}$ (i.e. $\mathbf{q}_{s} H_{0}^{1} \mathbf{q}_{t}$ or $\mathbf{q}_{s} H_{0}^{2} \mathbf{q}_{t}$ ). Rule (ii) captures transitivity. As for rule (iii), we note that the 'summed quantities' $\sum_{r \in \mathbf{r}} \mathbf{q}_{r}=\mathbf{q}_{t_{1}}+\mathbf{q}_{t_{2}}$ if $t_{1} \neq t_{2}$ and $\sum_{r \in \mathbf{r}} \mathbf{q}_{r}=\mathbf{q}_{t}$ if $t_{1}=t_{2}=t$. Given this, rule (iii) can again be interpreted in terms of Pareto efficiency. Specifically, it states that, if member $m$ prefers $\mathbf{q}_{t_{1}}$ over $\mathbf{q}_{s}$ for the bundle $\sum_{r \in \mathbf{r}} \mathbf{q}_{r}$ not more expensive than $\mathbf{q}_{s}$, then the choice of $\mathbf{q}_{s}$ can be rationalized only if the other member $l$ prefers $\mathbf{q}_{s}$ over $\mathbf{q}_{t_{2}}$. Indeed, if this last condition were not satisfied, then the bundle $\sum_{r \in \mathbf{r}} \mathbf{q}_{r}$ (under the given prices $\mathbf{p}_{s}$ and outlay $\mathbf{p}_{s}^{\prime} \mathbf{q}_{s}$ ) would imply a Pareto improvement over the chosen bundle $\mathbf{q}_{s}$. Rule (iv), finally, complements rule (iii). It states that, if member $m$ prefers $\mathbf{q}_{s_{m}}$ over $\mathbf{q}_{t}$, then the choice of $\mathbf{q}_{t}$ can be rationalized only if it is not more expensive than the (newly defined) 'summed quantities' $\sum_{r \in \mathbf{r}} \mathbf{q}_{r}$. Indeed, if this last condition were not met, then for the given prices $\mathbf{p}_{t}$ and outlay $\mathbf{p}_{t}^{\prime} \mathbf{q}_{t}$ both members would be better off by buying the quantities $\sum_{r \in \mathbf{r}} \mathbf{q}_{r}$ rather than the chosen quantities $\mathbf{q}_{t}$, which of course conflicts with collective rationality.

At this point, it is interesting to note that the $C A R P$ axiom has an analogous structure as the GARP axiom that applies to the unitary model. Specifically, $G A R P$ states (in casu unitary) rationality conditions in terms of 
the preference information that is revealed by the observed price and quantity data. Essentially, $C A R P$ does the same, but now the revealed preference information is understood in terms of the collective model of household consumption and, thus, pertains to the individual household members. ${ }^{5}$

We also recall that $G A R P$ provides a necessary and sufficient condition for rational consumption behavior in the context of the unitary model. The next theorem, which contains our core result, shows that $C A R P$ equally provides a necessary and sufficient condition for collectively rational consumption behavior. It provides a 'collective' version of the Afriat Theorem for unitary rationality.

Theorem 2 (Collective Afriat Theorem) Let $S=\left\{\left(\mathbf{p}_{t} ; \mathbf{q}_{t}\right) ; t=1, \ldots, T\right\}$ be a set of observations. The following statements are equivalent:

(i) There exists a pair of utility functions $U^{1}$ and $U^{2}$ that satisfy local collective non-satiation and that provide a collective rationalization of $S$;

(ii) The set $S$ satisfies CARP;

(iii) For all $t, r_{1}, r_{2} \in\{1, \ldots, T\}$, with $\mathbf{r}=\left\{r_{1}\right\} \cup\left\{r_{2}\right\}$, there exist numbers $U_{t}^{1}, U_{t}^{2}, \mu_{t \mathbf{r}}^{1}, \mu_{t \mathbf{r}}^{2} \in \mathbb{R}_{++}$that meet the collective Afriat inequalities

$$
\left[\mu_{t \mathbf{r}}^{1} U_{r_{1}}^{1}+\mu_{t \mathbf{r}}^{2} U_{r_{2}}^{2}\right]-\left[\mu_{t \mathbf{r}}^{1} U_{t}^{1}+\mu_{t \mathbf{r}}^{2} U_{t}^{2}\right] \leq \mathbf{p}_{t}^{\prime}\left(\sum_{r \in \mathbf{r}} \mathbf{q}_{r}-\mathbf{q}_{t}\right)
$$

(iv) There exists a pair of continuous and monotonically increasing utility functions $U^{1}$ and $U^{2}$ that satisfy local collective non-satiation and that provide a collective rationalization of $S$.

This result implies that data consistency with CARP is necessary and sufficient for a collective rationalization of the data; see condition (ii). In turn, this institutes a test for collective rationality that can be tested on the basis of observable price and quantity information. Next, condition (iii) provides an equivalent characterization in terms of collective Afriat inequalities; this complies with the Pareto efficiency characterization in Lemma 1 (using 'summed quantities' $\sum_{r \in \mathbf{r}} \mathbf{q}_{r}$ ). Finally, condition (iv) implies that, as

\footnotetext{
${ }^{5}$ In this respect, we can also refer to the discussion in Cherchye, De Rock, Vermeulen (2007, supplemental material). When presenting the generalized version of $C A R P$ for $M$-member households, the authors argue that $C A R P$ concides with GARP when $M=1$ (i.e. the household consists of a single decision maker/household member).
} 
soon as there exists a pair of utility functions that provide a rationalization, there exist continuous and monotone utility functions that provide a rationalization; in the current context, monotonicity means that $\widehat{\mathbf{q}}_{r_{1}} \geq \widehat{\mathbf{q}}_{r_{2}}$ and $\widehat{\mathbf{q}}_{r_{1}} \neq \widehat{\mathbf{q}}_{r_{2}}$ implies $U^{1}\left(\widehat{\mathbf{q}}_{r_{1}}\right) \geq U^{1}\left(\widehat{\mathbf{q}}_{r_{2}}\right)$ and $U^{2}\left(\widehat{\mathbf{q}}_{r_{1}}\right) \geq U^{2}\left(\widehat{\mathbf{q}}_{r_{2}}\right)$ with at least one strict inequality. Inter alia, this implies that for the general collective consumption model continuity and monotonicity are non-testable for a finite number of observations. Because a violation of monotonicity cannot be detected with a finite number of observations, we also conclude that the nature (positive or negative) of the consumption externalities is non-testable for the general consumption model. Section 6 , which relates our findings to existing results in the literature, will discuss additional interesting implications of the Collective Afriat Theorem. For example, it will turn out that concavity of the individual utility functions (representing convex preferences) is testable for the collective consumption model.

\section{Special cases}

In this section we discuss two special cases of the collective model: (i) the model with all consumption private and no externalities, which is also known as the 'egoistic' model, and (ii) the model in which all consumption is public. The next result states that, as soon as there exists a rationalization of the data in terms of the general collective consumption model, we can provide a rationalization of the same data in terms of the egoistic model.

Proposition 1 Suppose there exists a pair of utility functions $U^{1}$ and $U^{2}$ that satisfy local collective non-satiation and that provide a collective rationalization of $S$. Then, for $\mathbf{q}=\mathfrak{q}^{1}+\mathfrak{q}^{2}$, there exists a pair of continuous and monotonically increasing utility functions $U^{1}(\widehat{\mathbf{q}})=V^{1}\left(\mathfrak{q}^{1}\right)$ and $U^{2}(\widehat{\mathbf{q}})=V^{2}\left(\mathfrak{q}^{2}\right)$, which exclude consumption externalities and public consumption, that satisfy local collective non-satiation and that provide a collective rationalization of $S$.

We conclude that $C A R P$ consistency is also necessary and sufficient for a data rationalization in terms of the egoistic model, and that monotonicity is non-testable for this model. In addition, just like for the general model (see also our discussion in the next section), we also obtain for this special model that concavity of the individual utility functions is testable. 
It is interesting to compare the result in Proposition 1 with the following result, which pertains to the model with all consumption public.

Proposition 2 Suppose there exists a pair of utility functions $U^{1}$ and $U^{2}$ that satisfy local collective non-satiation and that provide a collective rationalization of $S$. Then, for $\mathbf{q}=\mathfrak{q}^{h}$, there exists a pair of continuous utility functions $U^{1}(\widehat{\mathbf{q}})=W^{1}\left(\mathfrak{q}^{h}\right)$ and $U^{2}(\widehat{\mathbf{q}})=W^{2}\left(\mathfrak{q}^{h}\right)$, which exclude private consumption, that satisfy local collective non-satiation and that provide a collective rationalization of $S$.

Thus, we again obtain that $C A R P$ provides a necessary and sufficient condition for data consistency with this special collective consumption model. The fact that $C A R P$ characterizes the general model as well as the two special models provides the general conclusion that, for the given setting that allows for possibly non-convex preferences, publicness or privateness of consumption does not yield testable implications; we will discuss this more elaborately in the next section. In addition, and analogous to before, we can conclude that concavity is testable for this special case with all consumption public. Still, contrary to before, it turns out that also monotonicity is testable for this special model. In other words, we may need utility functions with negative marginal utilities of the publicly consumed quantities to obtain a collective rationalization.

Example 1 in the Appendix illustrates the result on monotonicity: it presents data that require non-monotone individual utility functions for a rationalization in terms of the special model with all consumption public. In fact, the construction of the example also suggests an empirical test for the monotonicity property in this special case of the collective model. More specifically, there exist a pair of monotone utility functions $W^{1}$ and $W^{2}$ that provide a collective rationalization of the data (with only public consumption) if and only if the data satisfy $C A R P$ and, in addition to rules (i) to (iv) in Definition 6, the rule

$(\mathrm{v})$ if $\mathbf{q}_{s} \geq \mathbf{q}_{t}$ and $\mathbf{q}_{s} \neq \mathbf{q}_{t}$ then not $\mathbf{q}_{t} H^{1} \mathbf{q}_{s}$ and not $\mathbf{q}_{t} H^{2} \mathbf{q}_{s}$

Intuitively, we have $\mathbf{q}_{s}=\mathfrak{q}_{s}^{h}$ and $\mathbf{q}_{t}=\mathfrak{q}_{t}^{h}$ when all consumption is public, which means that all personalized quantity information is observed. And, thus, monotonicity excludes that any of the members prefers $\mathbf{q}_{t}$ over $\mathbf{q}_{s}$ when $\mathbf{q}_{s} \geq \mathbf{q}_{t}$ and $\mathbf{q}_{s} \neq \mathbf{q}_{t}$; this is captured by rule (v). We conclude that, if there do not exist relations $H_{0}^{m}$ and $H^{m}(m=1,2)$ that satisfy rules (i) to (iv) in 
Definition 6 and the above rule (v), then no monotonically increasing utility functions exist with only publicly consumed quantities as arguments.

\section{Relation to existing results}

Our results developed in the previous sections bear interesting relations with existing results. We can relate our nonparametric findings for the collective model to those on the (non)parametric characterization of collective consumption models under convex preferences. In addition, we can contrast our findings with those on the nonparametric characterization of the unitary model.

First, we can establish a relationship between our results and Chiappori and Ekeland's (2006) findings on the parametric characterization of collective consumption models while maintaining the assumption of convex preferences. As already indicated, we find that the same $C A R P$ condition is (necessary and sufficient) for data consistency with the general collective model as well as with the two special cases defined above. This parallels the conclusion of Chiappori and Ekeland that 'locally' (i.e. in a sufficiently small neighborhood of a given point) "an assumption like privateness (or publicness) of individual consumptions is not testable from data on group behavior" (Chiappori and Ekeland, 2006, p.4). In other words, Chiappori and Ekeland's 'local' parametric result for convex preferences complies with our, by construction 'global', nonparametric result when dropping the assumption of convex preferences. For completeness, we must add that the conclusion changes when requiring monotonicity of the data rationalizing utility functions: it follows from our results in the previous section that monotonicity is testable for the model with all consumption public, while it is non-testable for the other models under consideration (i.e. the general model and the model with all consumption private and no externalities).

It is also interesting to relate these conclusions to those of Cherchye, De Rock and Vermeulen (2008), who maintain the assumption of convex preferences, and who do obtain different nonparametric data consistency conditions for the different (i.e. general and special) collective consumption models. Notably, this finding of different 'global' (nonparametric) characterizations for different models contrasts with Chiappori and Ekeland's 'local' (parametric) conclusion cited above (-to recall- under the same convexity assumption). Cherchye, De Rock and Vermeulen interpret that these diverging 
findings confirm Chiappori and Ekeland's 'strong suspicion' that their local results must not hold globally. Following this interpretation, the results in the current paper add that Chiappori and Ekeland's conclusion does hold globally if convexity of the individual preferences is no longer maintained as an assumption.

Next, we can compare our results with those on the nonparametric characterization of the unitary consumption model (summarized by Varian, 1982 and 2006). In this respect, we first recall that the nonparametric characterization for the unitary model implies that monotonicity and concavity of a data rationalizing household utility function is not testable in the standard case with a linear budget set and positive prices. ${ }^{6}$ More specifically, it is well-known that, if there exists a household utility function that rationalizes the data in terms of the unitary model (because the data are consistent with $G A R P)$, then there always exists a monotone and concave household utility function that provides such a rationalization. See also Theorem 1 above.

In contrast to this conclusion for the unitary model, we find for the collective model that concavity of the individual household members' utility functions (or, convexity of the individual preferences) is testable. In particular, there may exist utility functions for the individual household members that rationalize the data in terms of the collective model (because the data are consistent with $C A R P$ ), while there do not exist concave individual utility functions that provide such a rationalization. This conclusion follows from contrasting the CARP condition, which characterizes the collective model when convex preferences are not imposed, with the condition in Proposition 1 of Cherchye, De Rock and Vermeulen (2007), which characterizes the collective model when convex preferences (implying a convex utility possibility set) are imposed. In general, the two conditions do not coincide. For instance, Example 2 of Cherchye, De Rock and Vermeulen (2007) provides data that pass $C A R P$ (which -to recall- these authors present as a necessary condition) but reject collective rationality under concave utility functions; these data can be rationalized in the collective consumption model, but not with concave individual utility functions.

Finally, as for monotonicity of the data rationalizing individual utility

\footnotetext{
${ }^{6}$ In the case of non-linear budget sets, Forges and Minelli (2006) argue that concavity is testable for the unitary model. Given our following argument, this a fortiori also holds for the collective model. In fact, our insights on testing data consistency with the collective model for linear budget sets could serve as a useful basis when considering non-linear budget sets in a collective setting.
} 
functions, we conclude that testability of monotonicity depends on the specific collective model under consideration. For the general model and the special model with all consumption private and no externalities (i.e. the egoistic model), we have demonstrated that monotonicity is non-testable when the data satisfy $C A R P$ : if there exist individual utility functions that rationalize the data in terms of these collective models, then there always exist monotone utility functions that provide such a rationalization. This implies that negative marginal utilities (including negative externalities) cannot be detected for these models. By contrast, we find that monotonicity of the individual utility functions can be rejected for the special model with no private consumption (i.e. all consumption is public). We conclude that, in this case, it is possible to detect negative marginal utilities of the publicly consumed goods. The intuition of the diverging results for the two special cases is the following. In the egoistic model, negative marginal utilities are pretty useless in the absence of externalities: individual rationality implies that goods with a negative impact on own utility are simply not chosen by the individual household member. This is not the case when all consumption is public. In that case, it cannot be ruled out that a certain public good has a positive impact on the utility of only one of the members, while it affects the other member negatively.

\section{Summary and conclusions}

We have presented a nonparametric 'revealed preference' characterization of the general model of collectively rational (i.e. Pareto efficient) household consumption behavior, which accounts for (positive or negative) externalities and public consumption in the household. Our distinguishing feature is that we allow for non-convex individual preferences. Given this, we have derived a necessary and sufficient condition for collective rationality. Because this condition provides a natural extension of Varian's Generalized Axiom of Revealed Preference (GARP) for individually rational consumption behavior, we have called it the Collective Axiom of Revealed Preference (CARP). Our main result provides a collective version of the Afriat Theorem for unitary rational behavior. We also obtain that, when accounting for possibly non-convex individual preferences, $C A R P$ characterizes not only the general model but also special cases of this general model (i.e. the case with all consumption private and no externalities, and the case with all consumption 
public).

In turn, our results provide some interesting testability conclusions, which bear relation to existing results on the (non)parametric characterization of collective consumption models under convex preferences, and on the nonparametric characterization of the unitary model. We find that, with only a finite set of observations, privateness (with or without externalities) or publicness of consumption is non-testable. For the general model and the special (egoistic) model with all consumption private and no externalities, the same non-testability conclusion holds for monotonicity of the individual utility functions; this also implies that, in the general model, the nature (positive or negative) of the consumption externalities cannot be tested. Still, monotonicity turns out to be testable for the special model with all consumption public. In addition, concavity of the individual utility functions (representing convex preferences) is testable for all collective consumption models under consideration.

Finally, our results suggest operational tests of collective rationality, and of concavity and monotonicity of the individual utility functions. More specifically, such tests can use the integer programming (IP) formulations in Cherchye, De Rock and Vermeulen (2008): these authors provide an IP test of the $C A R P$ condition, which -to recall- applies to collective rationality under possibly non-concave utility functions, as well as IP tests for collective rationality under concave utility functions. Comparison of the different test results allows us to conclude whether or not concave utility functions (representing convex preferences) rationalize the observed collective consumption behavior. An analogous IP formulation applies to the monotonicity test for the special model with all consumption public (suggested in Section 5). See Cherchye, De Rock, Sabbe and Vermeulen (2008) on the practical implementation of the IP tests.

\section{Appendix: proofs}

\section{Proof of Lemma 1}

Necessity. Suppose the utility functions $U^{1}$ and $U^{2}$ provide a collective rationalization of $S$. Because the result follows trivially if $U^{1}\left(\widehat{\mathbf{q}}_{t}\right) \geq U^{1}\left(\widehat{\mathbf{q}}_{r}\right)$ and $U^{2}\left(\widehat{\mathbf{q}}_{t}\right) \geq U^{2}\left(\widehat{\mathbf{q}}_{r}\right)$, we focus on $U^{m}\left(\widehat{\mathbf{q}}_{t}\right)>U^{m}\left(\widehat{\mathbf{q}}_{r}\right)$ and $U^{l}\left(\widehat{\mathbf{q}}_{t}\right)<U^{l}\left(\widehat{\mathbf{q}}_{r}\right)$ $(m \neq l)$. Then there exists $\mu_{t r}^{1}, \mu_{t r}^{2} \in \mathbb{R}_{++}$with $\frac{\mu_{t r}^{m}}{\mu_{t r}^{l}} \geq \frac{U^{l}\left(\widehat{\mathbf{q}}_{r}\right)-U^{l}\left(\widehat{\mathbf{q}}_{t}\right)}{U^{m}\left(\widehat{\mathbf{q}}_{t}\right)-U^{m}\left(\widehat{\mathbf{q}}_{r}\right)}>0$, 
which implies $\mu_{t r}^{1} U^{1}\left(\widehat{\mathbf{q}}_{t}\right)+\mu_{t r}^{2} U^{2}\left(\widehat{\mathbf{q}}_{t}\right) \geq \mu_{t r}^{1} U^{1}\left(\widehat{\mathbf{q}}_{r}\right)+\mu_{t r}^{2} U^{2}\left(\widehat{\mathbf{q}}_{r}\right)$.

Sufficiency. Suppose there exist utility functions $U^{1}$ and $U^{2}$ and $\mu_{t r}^{1}, \mu_{t r}^{2} \in$ $\mathbb{R}_{++}$that imply, for each $t, \mu_{t r}^{1} U^{1}\left(\widehat{\mathbf{q}}_{t}\right)+\mu_{t r}^{2} U^{2}\left(\widehat{\mathbf{q}}_{t}\right) \geq \mu_{t r}^{1} U^{1}\left(\widehat{\mathbf{q}}_{r}\right)+\mu_{t r}^{2} U^{2}\left(\widehat{\mathbf{q}}_{r}\right)$ for some feasible personalized quantities $\widehat{\mathbf{q}}_{t}$ and all $\widehat{\mathbf{q}}_{r}$ with $\mathbf{p}_{t}^{\prime} \mathbf{q}_{r} \leq \mathbf{p}_{t}^{\prime} \mathbf{q}_{t}$. We prove ad absurdum. Suppose the functions $U^{1}$ and $U^{2}$ do not provide a collective rationalization of $S$. That is, for some $t$ we have for all $\widehat{\mathbf{q}}_{t}$ that there exists $\widehat{\mathbf{q}}_{r}$ such that $U^{m}\left(\widehat{\mathbf{q}}_{t}\right)<U^{m}\left(\widehat{\mathbf{q}}_{r}\right)$ and $U^{l}\left(\widehat{\mathbf{q}}_{t}\right) \leq U^{l}\left(\widehat{\mathbf{q}}_{r}\right)$. But then, for the given $t$, we have for all $\widehat{\mathbf{q}}_{t}$ that there exists $\widehat{\mathbf{q}}_{r}$ such that $\mu_{t r}^{1} U^{1}\left(\widehat{\mathbf{q}}_{t}\right)+\mu_{t r}^{2} U^{2}\left(\widehat{\mathbf{q}}_{t}\right)<\mu_{t r}^{1} U^{1}\left(\widehat{\mathbf{q}}_{r}\right)+\mu_{t r}^{2} U^{2}\left(\widehat{\mathbf{q}}_{r}\right)$ for all $\mu_{t r}^{1}, \mu_{t r}^{2} \in \mathbb{R}_{++}$; and this implies a contradiction.

\section{Proof of Theorem 2}

Step 1: (i) implies (ii). Suppose there exists a pair of collectively nonsatiated utility functions $U^{1}$ and $U^{2}$ that provide a collective rationalization of $S$. This implies that for all $t \in\{1, \ldots, T\}$ there exists a specification of the feasible personalized quantities $\widehat{\mathbf{q}}_{t}$ such that $U^{m}\left(\widehat{\mathbf{q}}_{r}\right)>U^{m}\left(\widehat{\mathbf{q}}_{t}\right)$ implies $U^{l}\left(\widehat{\mathbf{q}}_{r}\right)<U^{l}\left(\widehat{\mathbf{q}}_{t}\right)(m \neq l)$ for all $\widehat{\mathbf{q}}_{r}$ with $\mathbf{p}_{t}^{\prime} \mathbf{q}_{r} \leq \mathbf{p}_{t}^{\prime} \mathbf{q}_{t}$.

For this specification of the feasible personalized quantities, we can specify hypothetical relations $H^{m}$ for all $s \in\{1, \ldots, T\}$ and $m \in\{1,2\}$ as follows:

$$
\mathbf{q}_{s} H^{m} \mathbf{q}_{t} \Leftrightarrow U^{m}\left(\widehat{\mathbf{q}}_{s}\right) \geq U^{m}\left(\widehat{\mathbf{q}}_{t}\right)
$$

We next have to verify whether this specification of the hypothetical relations satisfies the rules (i)-(iv) in the $C A R P$ Definition 6 . Note that we do not distinguish between the relations $H^{m}$ and $H_{0}^{m}$ because the specification of the relation $H^{m}$ is obtained by using real numbers. This also makes that rule (ii) is automatically satisfied.

As for rule (i), a collective rationalization of the data requires that $\mathbf{p}_{s}^{\prime} \mathbf{q}_{s} \geq$ $\mathbf{p}_{s}^{\prime} \mathbf{q}_{t}$ implies that for any $\widehat{\mathbf{q}}_{s}$ and $\widehat{\mathbf{q}}_{t}$ we have $U^{1}\left(\widehat{\mathbf{q}}_{s}\right) \geq U^{1}\left(\widehat{\mathbf{q}}_{t}\right)$ or $U^{2}\left(\widehat{\mathbf{q}}_{s}\right) \geq$ $U^{2}\left(\widehat{\mathbf{q}}_{t}\right)$, which necessarily obtains $\mathbf{q}_{s} H^{1} \mathbf{q}_{t}$ or $\mathbf{q}_{s} H^{2} \mathbf{q}_{t}$.

As for rule (iii), we make the distinction between $t_{1}=t_{2}=t$, which implies $\mathbf{r}=\{t\}$, and $t_{1} \neq t_{2}$, which implies $\mathbf{r}=\left\{t_{1}, t_{2}\right\}$. We first consider $\mathbf{r}=\{t\}$, and we prove rule (iii) ad absurdum. Suppose $\mathbf{p}_{s}^{\prime} \mathbf{q}_{s} \geq \mathbf{p}_{s}^{\prime} \mathbf{q}_{t}$ in combination with $\mathbf{q}_{t} H^{m} \mathbf{q}_{s}$ and not $\mathbf{q}_{s} H^{l} \mathbf{q}_{t}$ (with $l \neq m$ ). Given our specification of the hypothetical relations, this implies that $U^{m}\left(\widehat{\mathbf{q}}_{t}\right) \geq U^{m}\left(\widehat{\mathbf{q}}_{s}\right)$ and $U^{l}\left(\widehat{\mathbf{q}}_{s}\right)<U^{l}\left(\widehat{\mathbf{q}}_{t}\right)$. But then $\widehat{\mathbf{q}}_{s}$ is not Pareto efficient, which conflicts with a collective rationalization of the data. 
We use local collective non-satiation to obtain that rule (iii) is satisfied for $\mathbf{r}=\left\{t_{1}, t_{2}\right\}$. To see this, suppose rule (iii) does not hold, i.e. $\mathbf{q}_{t_{1}} H^{m} \mathbf{q}_{s}$ and not $\mathbf{q}_{s} H^{l} \mathbf{q}_{t_{2}}($ with $l \neq m)$ for $\mathbf{p}_{s}^{\prime} \mathbf{q}_{s} \geq \mathbf{p}_{s}^{\prime}\left(\mathbf{q}_{t_{1}}+\mathbf{q}_{t_{2}}\right)$. Note that $\mathbf{q}_{t_{1}} H^{m} \mathbf{q}_{s}$ and not $\mathbf{q}_{s} H^{l} \mathbf{q}_{t_{2}}$ implies that $U^{m}\left(\widehat{\mathbf{q}}_{t_{1}}\right) \geq U^{m}\left(\widehat{\mathbf{q}}_{s}\right)$ and $U^{l}\left(\widehat{\mathbf{q}}_{s}\right)<U^{l}\left(\widehat{\mathbf{q}}_{t_{2}}\right)$. Local collective non-satiation implies that for any $\epsilon \geq 0$ there exist quantities $\mathbf{q}$ with $\left\|\mathbf{q}_{t_{1}}+\mathbf{q}_{t_{2}}-\mathbf{q}\right\| \leq \epsilon$ for which $\mathbf{p}_{s}^{\prime} \mathbf{q}_{s} \geq \mathbf{p}_{s}^{\prime} \mathbf{q}$ and such that $U^{m}(\widehat{\mathbf{q}}) \geq$ $U^{m}\left(\widehat{\mathbf{q}}_{t_{1}}\right)$ and $U^{l}(\widehat{\mathbf{q}}) \geq U^{l}\left(\widehat{\mathbf{q}}_{t_{2}}\right)$. But then, given the above, we also have that $U^{m}(\widehat{\mathbf{q}}) \geq U^{m}\left(\widehat{\mathbf{q}}_{s}\right)$ and $U^{l}(\widehat{\mathbf{q}})>U^{l}\left(\widehat{\mathbf{q}}_{s}\right)$, and $\mathbf{p}_{s}^{\prime} \mathbf{q}_{s} \geq \mathbf{p}_{s}^{\prime} \mathbf{q}$; this means that $\widehat{\mathbf{q}}_{s}$ is not Pareto efficient, which conflicts with a collective rationalization of the set $S$.

As for rule (iv), we again make the distinction between $s_{1}=s_{2}=s$, which implies $\mathbf{r}=\{s\}$, and $s_{1} \neq s_{2}$, which implies $\mathbf{r}=\left\{s_{1}, s_{2}\right\}$. We first consider $\mathbf{r}=\{s\}$, and we prove rule (iv) ad absurdum. Suppose $\mathbf{q}_{s} H^{1} \mathbf{q}_{t}$ and $\mathbf{q}_{s} H^{2} \mathbf{q}_{t}$ in combination with $\mathbf{p}_{t}^{\prime} \mathbf{q}_{t}>\mathbf{p}_{t}^{\prime} \mathbf{q}_{s}$. On the one hand, $\mathbf{q}_{s} H^{1}$ $\mathbf{q}_{t}$ and $\mathbf{q}_{s} H^{2} \mathbf{q}_{t}$ implies $U^{1}\left(\widehat{\mathbf{q}}_{s}\right) \geq U^{1}\left(\widehat{\mathbf{q}}_{t}\right)$ and $U^{2}\left(\widehat{\mathbf{q}}_{s}\right) \geq U^{2}\left(\widehat{\mathbf{q}}_{t}\right)$. On the other hand, because $\mathbf{p}_{t}^{\prime} \mathbf{q}_{t}>\mathbf{p}_{t}^{\prime} \mathbf{q}_{s}$, local collective non-satiation implies that there exists $\mathbf{q}$ close enough to the quantities $\mathbf{q}_{s}$ with $\mathbf{p}_{t}^{\prime} \mathbf{q}_{t}>\mathbf{p}_{t}^{\prime} \mathbf{q}$ so that $U^{m}(\widehat{\mathbf{q}}) \geq U^{m}\left(\widehat{\mathbf{q}}_{s}\right)$ and $U^{l}(\widehat{\mathbf{q}}) \geq U^{l}\left(\widehat{\mathbf{q}}_{s}\right)$ with at least one strict inequality. But then $U^{m}(\widehat{\mathbf{q}}) \geq U^{m}\left(\widehat{\mathbf{q}}_{t}\right)$ and $U^{l}(\widehat{\mathbf{q}}) \geq U^{l}\left(\widehat{\mathbf{q}}_{t}\right)$, with at least one strict inequality, and $\mathbf{p}_{t}^{\prime} \mathbf{q}_{t}>\mathbf{p}_{t}^{\prime} \mathbf{q}$; this means that $\widehat{\mathbf{q}}_{t}$ is not Pareto efficient, which conflicts with a collective rationalization of the set $S$.

We analogously prove that rule (iv) is satisfied for $\mathbf{r}=\left\{s_{1}, s_{2}\right\}$. For $s_{1} \neq$ $s_{2}$, let $\mathbf{q}_{s_{1}} H^{1} \mathbf{q}_{t}$ and $\mathbf{q}_{s_{2}} H^{2} \mathbf{q}_{t}$ in combination with $\mathbf{p}_{t}^{\prime} \mathbf{q}_{t}>\mathbf{p}_{t}^{\prime}\left(\mathbf{q}_{s_{1}}+\mathbf{q}_{s_{2}}\right)$. On the one hand, $\mathbf{q}_{s_{1}} H^{1} \mathbf{q}_{t}$ and $\mathbf{q}_{s_{2}} H^{2} \mathbf{q}_{t}$ implies $U^{1}\left(\widehat{\mathbf{q}}_{s_{1}}\right) \geq U^{1}\left(\widehat{\mathbf{q}}_{t}\right)$ and $U^{2}\left(\widehat{\mathbf{q}}_{s_{2}}\right) \geq U^{2}\left(\widehat{\mathbf{q}}_{t}\right)$. On the other hand, because $\mathbf{p}_{t}^{\prime} \mathbf{q}_{t}>\mathbf{p}_{t}^{\prime}\left(\mathbf{q}_{s_{1}}+\mathbf{q}_{s_{2}}\right)$, one can analogously as in rule (iii) show that local collective non-satiation implies that $\widehat{\mathbf{q}}_{t}$ is not Pareto efficient, which conflicts with a collective rationalization of the set $S$.

Step 2: (ii) implies (iii). We must show that, if the set $S$ satisfies $C A R P$, then for all $t, r_{1}, r_{2} \in\{1, \ldots, T\}$, with $\mathbf{r}=\left\{r_{1}\right\} \cup\left\{r_{2}\right\}$, there exist numbers $U_{t}^{1}, U_{t}^{2}, \mu_{t \mathbf{r}}^{1}, \mu_{t \mathbf{r}}^{2} \in \mathbb{R}_{++}$that meet the collective Afriat inequalities.

First, for the given specification of the relations $H^{m}$ consistent with $C A R P$, we specify the numbers $U_{t}^{m} \in \mathbb{R}_{++}$that satisfy for each $\mathbf{q}_{t_{1}}, \mathbf{q}_{t_{2}}$ :

$$
\begin{aligned}
& \text { if } \mathbf{q}_{t_{1}} H^{m} \mathbf{q}_{t_{2}} \text { and not } \mathbf{q}_{t_{2}} H^{m} \mathbf{q}_{t_{1}} \text { then } U_{t_{1}}^{m}>U_{t_{2}}^{m} ; \\
& \text { and if } \mathbf{q}_{t_{1}} H^{m} \mathbf{q}_{t_{2}} \text { and } \mathbf{q}_{t_{2}} H^{m} \mathbf{q}_{t_{1}} \text { then } U_{t_{1}}^{m}=U_{t_{2}}^{m} .
\end{aligned}
$$

Note that, if not $\mathbf{q}_{t_{1}} H^{m} \mathbf{q}_{t_{2}}$ and not $\mathbf{q}_{t_{2}} H^{m} \mathbf{q}_{t_{1}}$, then there is no restriction on 
the corresponding values of $U_{t_{1}}^{m}$ and $U_{t_{2}}^{m}$. Generally, the specific values of $U_{t_{1}}^{m}$ and $U_{t_{2}}^{m}$ are irrelevant for our following argument.

Given this, for each $t, r_{1}, r_{2} \in\{1, \ldots, T\}$, with $\mathbf{r}=\left\{r_{1}\right\} \cup\left\{r_{2}\right\}$, we must specify numbers $\mu_{t \mathbf{r}}^{1}, \mu_{t \mathbf{r}}^{2} \in \mathbb{R}_{++}$such that the corresponding collective Afriat inequality $\left[\mu_{t \mathbf{r}}^{1} U_{r_{1}}^{1}+\mu_{t \mathbf{r}}^{2} U_{r_{2}}^{2}\right]-\left[\mu_{t \mathbf{r}}^{1} U_{t}^{1}+\mu_{t \mathbf{r}}^{2} U_{t}^{2}\right] \leq \mathbf{p}_{t}^{\prime}\left(\sum_{r \in \mathbf{r}} \mathbf{q}_{r}-\mathbf{q}_{t}\right)$ is met. We distinguish two possible cases:

CASE 1: $\mathbf{q}_{r_{1}} H^{1} \mathbf{q}_{t}$ and $\mathbf{q}_{r_{2}} H^{2} \mathbf{q}_{t}$. Given the specification of the numbers $U_{t}^{m}$ in (2) and (3), we then have that $U_{r_{1}}^{1} \geq U_{t}^{1}$ and $U_{r_{2}}^{2} \geq U_{t}^{2}$. Since $\mathbf{q}_{r_{1}} H^{1} \mathbf{q}_{t}$ and $\mathbf{q}_{r_{2}} H^{2} \mathbf{q}_{t}$, rule (iv) of Definition 6 implies that we must have $\mathbf{p}_{t}^{\prime}\left(\sum_{r \in \mathbf{r}} \mathbf{q}_{r}\right.$ $\left.-\mathbf{q}_{t}\right) \geq 0$. For $\mathbf{p}_{t}^{\prime}\left(\sum_{r \in \mathbf{r}} \mathbf{q}_{r}-\mathbf{q}_{t}\right)>0$ the corresponding collective Afriat inequality is satisfied by setting $\mu_{t \mathbf{r}}^{1}$ and $\mu_{t \mathbf{r}}^{2}$ sufficiently small. Next, for $\mathbf{p}_{t}^{\prime}\left(\sum_{r \in \mathbf{r}} \mathbf{q}_{r}-\mathbf{q}_{t}\right)=0$ we note that $\mathbf{q}_{r_{1}} H^{1} \mathbf{q}_{t}$ and $\mathbf{q}_{r_{2}} H^{2} \mathbf{q}_{t}$ imply, respectively, $\mathbf{q}_{t} H^{2} \mathbf{q}_{r_{2}}$ and $\mathbf{q}_{t} H^{1} \mathbf{q}_{r_{1}}$ (see rule (iii) in Definition 6). As a result, we obtain $U_{r_{1}}^{1}=U_{t}^{1}$ and $U_{r_{2}}^{2}=U_{t}^{2}$ because of (3), and the corresponding collective Afriat inequality is satisfied for any $\mu_{t \mathbf{r}}^{1}$ and $\mu_{t \mathbf{r}}^{2}$.

CASE 2: Not $\mathbf{q}_{r_{1}} H^{1} \mathbf{q}_{t}$ or not $\mathbf{q}_{r_{2}} H^{2} \mathbf{q}_{t}$. Without losing generality, let us assume that we have not $\mathbf{q}_{r_{1}} H^{1} \mathbf{q}_{t}$. Our specification in (2) and (3) then implies that we exclude $U_{r_{1}}^{1} \geq U_{t}^{1}$. So we can specify that $U_{r_{1}}^{1}<U_{t}^{1}$. For the given value of $\mathbf{p}_{t}^{\prime}\left(\sum_{r \in \mathbf{r}} \mathbf{q}_{r}-\mathbf{q}_{t}\right)$, we then set $\mu_{t \mathbf{r}}^{1}$ sufficiently large and $\mu_{t \mathbf{r}}^{2}$ sufficiently small such that $\mu_{t \mathbf{r}}^{1}\left[U_{r_{1}}^{1}-U_{t}^{1}\right] \leq \mathbf{p}_{t}^{\prime}\left(\sum_{r \in \mathbf{r}} \mathbf{q}_{r}-\mathbf{q}_{t}\right)-\mu_{t \mathbf{r}}^{2}\left[U_{r_{2}}^{2}-U_{t}^{2}\right]$, i.e. the corresponding collective Afriat inequality is met.

Step 3: (iii) implies (iv). Our proof contains two steps. We address data rationalization in Step 3a, and continuity and monotonicity in Step 3b.

Step 3a: data rationalization. Suppose for all $t, r_{1}, r_{2} \in\{1, \ldots, T\}$, with $\mathbf{r}=\left\{r_{1}\right\} \cup\left\{r_{2}\right\}$, there exist numbers $U_{t}^{1}, U_{t}^{2}, \mu_{t \mathbf{r}}^{1}, \mu_{t \mathbf{r}}^{2} \in \mathbb{R}_{++}$that meet the collective Afriat inequalities. We have to prove that we can then specify utility functions $U^{1}$ and $U^{2}$ such that for each observation $t$ there exist feasible personalized quantities $\widehat{\mathbf{q}}_{t}$ and, for all $\widehat{\mathbf{q}}_{r}$ with $\mathbf{p}_{t}^{\prime} \mathbf{q}_{r} \leq \mathbf{p}_{t}^{\prime} \mathbf{q}_{t}, \mu^{1}, \mu^{2} \in \mathbb{R}_{++}$ that imply

$$
\mu^{1} U^{1}\left(\widehat{\mathbf{q}}_{t}\right)+\mu^{2} U^{2}\left(\widehat{\mathbf{q}}_{t}\right) \geq \mu^{1} U^{1}\left(\widehat{\mathbf{q}}_{r}\right)+\mu^{2} U^{2}\left(\widehat{\mathbf{q}}_{r}\right) .
$$

To obtain the result, we define $U^{1}\left(\widehat{\mathbf{q}}_{r}\right)$ and $U^{2}\left(\widehat{\mathbf{q}}_{r}\right)$ such that

$$
\begin{aligned}
U^{1}\left(\widehat{\mathbf{q}}_{r}\right) & =\max _{t} \min _{s}\left[U_{t}^{1}+\frac{\mathbf{p}_{s}^{\prime}\left(\mathbf{q}_{r}-\mathbf{q}_{t}\right)}{\mu_{s\{t\}}^{1}} \text { subject to } U_{t}^{2} \geq \bar{U}_{r}^{2}\right], \text { and } \\
U^{2}\left(\widehat{\mathbf{q}}_{r}\right) & =U_{t^{*}}^{2},
\end{aligned}
$$


with $t^{*}$ the observation that solves the max problem in (4). As for the specification of $\bar{U}_{r}^{2}$, we use:

if $r$ is observed then $\bar{U}_{r}^{2}=U_{r}^{2}$,

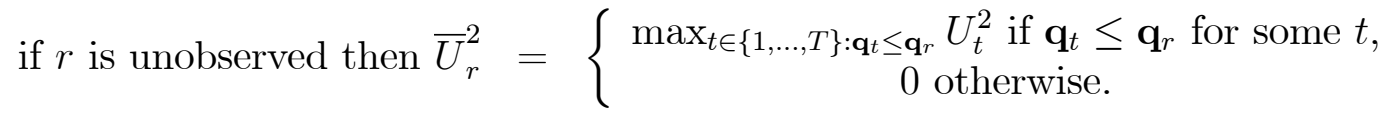

As a first step, we show that we have $U^{1}\left(\widehat{\mathbf{q}}_{t}\right)=U_{t}^{1}$ and $U^{2}\left(\widehat{\mathbf{q}}_{t}\right)=U_{t}^{2}$ for each observed $t$. We prove ad absurdum. If the result does not hold, then there exists $t^{*} \neq t$ such that

$$
U_{t}^{1}<U_{t^{*}}^{1}+\min _{s} \frac{\mathbf{p}_{s}^{\prime}\left(\mathbf{q}_{t}-\mathbf{q}_{t^{*}}\right)}{\mu_{s\left\{t^{*}\right\}}^{1}} \text { and } U_{t}^{2} \leq U_{t^{*}}^{2}
$$

In that case, we have for all $\mu^{1} \in \mathbb{R}_{++}$and $\mu^{2} \in \mathbb{R}_{++}$:

$$
\mu^{1} U_{t}^{1}+\mu^{2} U_{t}^{2}<\mu^{1}\left[U_{t^{*}}^{1}+\min _{s} \frac{\mathbf{p}_{s}^{\prime}\left(\mathbf{q}_{t}-\mathbf{q}_{t^{*}}\right)}{\mu_{s\left\{t^{*}\right\}}^{1}}\right]+\mu^{2} U_{t^{*}}^{2},
$$

and thus

$$
\mu^{1} U_{t}^{1}+\mu^{2} U_{t}^{2}<\mu^{1} U_{t^{*}}^{1}+\mu^{2} U_{t^{*}}^{2}+\mu^{1}\left[\frac{\mathbf{p}_{t}^{\prime}\left(\mathbf{q}_{t}-\mathbf{q}_{t^{*}}\right)}{\mu_{t\left\{t^{*}\right\}}^{1}}\right]
$$

Now consider $\mu^{1}=\mu_{t\left\{t^{*}\right\}}^{1}, \mu^{2}=\mu_{t\left\{t^{*}\right\}}^{2}$. We obtain

$$
\mu_{t\left\{t^{*}\right\}}^{1} U_{t}^{1}+\mu_{t\left\{t^{*}\right\}}^{2} U_{t}^{2}<\mu_{t\left\{t^{*}\right\}}^{1} U_{t^{*}}^{1}+\mu_{t\left\{t^{*}\right\}}^{2} U_{t^{*}}^{2}+\mathbf{p}_{t}^{\prime}\left(\mathbf{q}_{t}-\mathbf{q}_{t^{*}}\right)
$$

or

$$
\mathbf{p}_{t}^{\prime}\left(\mathbf{q}_{t^{*}}-\mathbf{q}_{t}\right)<\left(\mu_{t\left\{t^{*}\right\}}^{1} U_{t^{*}}^{1}+\mu_{t\left\{t^{*}\right\}}^{2} U_{t^{*}}^{2}\right)-\left(\mu_{t\left\{t^{*}\right\}}^{1} U_{t}^{1}+\mu_{t\left\{t^{*}\right\}}^{2} U_{t}^{2}\right)
$$

which violates the collective Afriat inequalities.

Next, we show that our specification of $U^{1}$ and $U^{2}$ provides a collective rationalization of the set $S$. Consider $\mathbf{q}_{r}$ and $\mathbf{p}_{t}^{\prime} \mathbf{q}_{t} \geq \mathbf{p}_{t}^{\prime} \mathbf{q}_{r}$ for some observed $t$. Then we must show that there exist $\mu^{1}, \mu^{2} \in \mathbb{R}_{++}$such that $\mu^{1} U^{1}\left(\widehat{\mathbf{q}}_{t}\right)+$ $\mu^{2} U^{2}\left(\widehat{\mathbf{q}}_{t}\right) \geq \mu^{1} U^{1}\left(\widehat{\mathbf{q}}_{r}\right)+\mu^{2} U^{2}\left(\widehat{\mathbf{q}}_{r}\right)$, with $U^{1}\left(\widehat{\mathbf{q}}_{r}\right)$ and $U^{2}\left(\widehat{\mathbf{q}}_{r}\right)$ defined in (4) and (5). We prove the result for $\mu^{1}=\mu_{t\left\{t^{*}\right\}}^{1}$ and $\mu^{2}=\mu_{t\left\{t^{*}\right\}}^{2}$. First, because of the collective Afriat inequalities we have

$$
\left[\mu_{t\left\{t^{*}\right\}}^{1} U_{t^{*}}^{1}+\mu_{t\left\{t^{*}\right\}}^{2} U_{t^{*}}^{2}\right]-\left[\mu_{t\left\{t^{*}\right\}}^{1} U_{t}^{1}+\mu_{t\left\{t^{*}\right\}}^{2} U_{t}^{2}\right] \leq \mathbf{p}_{t}^{\prime}\left(\mathbf{q}_{t^{*}}-\mathbf{q}_{t}\right)
$$


which implies

$$
\begin{aligned}
\mu_{t\left\{t^{*}\right\}}^{1} U_{t^{*}}^{1}+\mu_{t\left\{t^{*}\right\}}^{2} U_{t^{*}}^{2}+\mathbf{p}_{t}^{\prime}\left(\mathbf{q}_{r}-\mathbf{q}_{t^{*}}\right) & \leq \mu_{t\left\{t^{*}\right\}}^{1} U_{t}^{1}+\mu_{t\left\{t^{*}\right\}}^{2} U_{t}^{2}+\mathbf{p}_{t}^{\prime}\left(\mathbf{q}_{r}-\mathbf{q}_{t}\right) \\
& \leq \mu_{t\left\{t^{*}\right\}}^{1} U_{t}^{1}+\mu_{t\left\{t^{*}\right\}}^{2} U_{t}^{2} \\
& =\mu_{t\left\{t^{*}\right\}}^{1} U^{1}\left(\widehat{\mathbf{q}}_{t}\right)+\mu_{t\left\{t^{*}\right\}}^{2} U^{2}\left(\widehat{\mathbf{q}}_{t}\right) .
\end{aligned}
$$

It suffices then to show that

$$
\mu_{t\left\{t^{*}\right\}}^{1} U^{1}\left(\widehat{\mathbf{q}}_{r}\right)+\mu_{t\left\{t^{*}\right\}}^{2} U^{2}\left(\widehat{\mathbf{q}}_{r}\right) \leq \mu_{t\left\{t^{*}\right\}}^{1} U_{t^{*}}^{1}+\mu_{t\left\{t^{*}\right\}}^{2} U_{t^{*}}^{2}+\mathbf{p}_{t}^{\prime}\left(\mathbf{q}_{r}-\mathbf{q}_{t^{*}}\right)
$$

or, using (4) and (5),

$\mu_{t\left\{t^{*}\right\}}^{1} U_{t^{*}}^{1}+\mu_{t\left\{t^{*}\right\}}^{2} U_{t^{*}}^{2}+\mu_{t\left\{t^{*}\right\}}^{1}\left[\frac{\mathbf{p}_{s^{*}}^{\prime}\left(\mathbf{q}_{r}-\mathbf{q}_{t^{*}}\right)}{\mu_{s^{*}\left\{t^{*}\right\}}^{1}}\right] \leq \mu_{t\left\{t^{*}\right\}}^{1} U_{t^{*}}^{1}+\mu_{t\left\{t^{*}\right\}}^{2} U_{t^{*}}^{2}+\mathbf{p}_{t}^{\prime}\left(\mathbf{q}_{r}-\mathbf{q}_{t^{*}}\right)$,

with $s^{*}$ the observation that solves the min problem in (4). The resulting condition

$$
\frac{\mathbf{p}_{s^{*}}^{\prime}\left(\mathbf{q}_{r}-\mathbf{q}_{t^{*}}\right)}{\mu_{s^{*}\left\{t^{*}\right\}}^{1}} \leq \frac{\mathbf{p}_{t}^{\prime}\left(\mathbf{q}_{r}-\mathbf{q}_{t^{*}}\right)}{\mu_{t\left\{t^{*}\right\}}^{1}}
$$

is satisfied because $s^{*}$ solves the min problem in (4). We obtain that there exists a pair of utility functions $U^{1}$ and $U^{2}$ that provide a collective rationalization of $S$.

Step 3b: monotonicity and continuity. Given the construction of $U^{1}$ and $U^{2}$ in (4) and (5), we can always construct $\widehat{\mathbf{q}}$ that obtains monotonicity and continuity. Let us first consider monotonicity. For observed $t_{1}$ and $t_{2}$, monotonicity means that $\widehat{\mathbf{q}}_{t_{1}} \geq \widehat{\mathbf{q}}_{t_{2}}$ and $\widehat{\mathbf{q}}_{t_{1}} \neq \widehat{\mathbf{q}}_{t_{2}}$ implies $U^{1}\left(\widehat{\mathbf{q}}_{t_{1}}\right) \geq$ $U^{1}\left(\widehat{\mathbf{q}}_{t_{2}}\right)$ and $U^{2}\left(\widehat{\mathbf{q}}_{t_{1}}\right) \geq U^{2}\left(\widehat{\mathbf{q}}_{t_{2}}\right)$, with at least one strict inequality. This condition is easily met for a given set $S$. For example, suppose that there are 2 goods $e_{1}, e_{2} \in\{1, \ldots, N\}$ with strictly positive quantities for all observed $t$, i.e. $\left(\mathbf{q}_{t}\right)_{e_{1}}>0$ and $\left(\mathbf{q}_{t}\right)_{e_{2}}>0$ for all $t$ (with $(\mathbf{x})_{e}$ the $e$-th entry of the vector $\mathbf{x})$. In that case, it suffices to specify $\left(\mathfrak{q}_{t_{1}}^{m}\right)_{e_{m}}>\left(\mathfrak{q}_{t_{2}}^{m}\right)_{e_{m}}$ if $U^{m}\left(\widehat{\mathbf{q}}_{t_{1}}\right)>$ $U^{m}\left(\widehat{\mathbf{q}}_{t_{2}}\right)(m=1,2)$. (Empirically, the existence of $e_{1}$ and $e_{2}$ with $\left(\mathbf{q}_{t}\right)_{e_{1}}>$ 0 and $\left(\mathbf{q}_{t}\right)_{e_{2}}>0$ for all observed $t$ is a very mild assumption. Formally similar constructions are possible if this assumption is not met, but they are mathematically less elegant.) Using (4) and (5), monotonicity in terms of $\widehat{\mathbf{q}}_{t}$ and $U^{m}\left(\widehat{\mathbf{q}}_{t}\right)$ for observed $t$ implies monotonicity in terms of $\widehat{\mathbf{q}}_{r}$ with $\mathbf{q}_{r}$ observed or unobserved. 
Let us then consider continuity. If the functions $U^{1}$ and $U^{2}$ in (4) and (5) have any discontinuities, their number will be finite by construction, because the number of observations $T$ is finite. As such, the discontinuities can be 'fixed' by linear interpolation without interfering with the rationalization argument in Step 3a.

Step 4: (iv) implies (i). This is trivial.

\section{Proof of Propositions 1 and 2}

The results follow from the construction of the proof of Theorem 2. Specifically, the data rationalization argument (Step 3a) and the continuity argument (using linear interpolation; Step 3b) apply for general utility functions $U^{1}$ and $U^{2}$ defined in general $\widehat{\mathbf{q}}$. These arguments directly extend to the special cases with utility functions $V^{m}$ defined in $\mathfrak{q}^{m}(m=1,2)$, for Proposition 1 , and utility functions $W^{m}$ defined in $\mathfrak{q}^{h}(m=1,2)$, for Proposition 2. Finally, the argument for monotonicity (Step $3 \mathrm{~b}$ of the proof of Theorem 2) directly extends to the case with utility functions $V^{m}$ defined in $\mathfrak{q}^{m}$ $(m=1,2)$, which completes the proof of Proposition 1 .

\section{Example 1}

This example illustrates that monotonicity of the individual utility functions is testable when private consumption is excluded (i.e. all consumption is public). As a preliminary remark, we recall that $W^{m}\left(\mathbf{q}_{t}\right)=U_{t}^{m}$ for observed $t$. We thus need a set $S$ that satisfies CARP only if $\mathbf{q}_{t_{2}} H^{m} \mathbf{q}_{t_{1}}$ (and not $\mathbf{q}_{t_{1}} H^{m} \mathbf{q}_{t_{2}}$ ) for $\mathbf{q}_{t_{1}} \geq \mathbf{q}_{t_{2}}$ and $\mathbf{q}_{t_{1}} \neq \mathbf{q}_{t_{2}}$ : using (1), this corresponds to $W^{m}\left(\mathbf{q}_{t_{2}}\right)>W^{m}\left(\mathbf{q}_{t_{1}}\right)$ for $\mathbf{q}_{t_{1}} \geq \mathbf{q}_{t_{2}}$ and $\mathbf{q}_{t_{1}} \neq \mathbf{q}_{t_{2}}$, which violates monotonicity. We complete the argument by considering the set $S=\left\{\left(\mathbf{p}_{t} ; \mathbf{q}_{t}\right), t=1,2,3,4\right\}$ with

$$
\begin{aligned}
& \mathbf{q}_{1}=\left(\begin{array}{l}
2 \\
1 \\
0
\end{array}\right), \mathbf{q}_{2}=\left(\begin{array}{l}
0 \\
0 \\
1
\end{array}\right), \mathbf{q}_{3}=\left(\begin{array}{l}
1 \\
2 \\
0
\end{array}\right), \mathbf{q}_{4}=\left(\begin{array}{l}
1 \\
1 \\
0
\end{array}\right) ; \\
& \mathbf{p}_{1}=\left(\begin{array}{l}
5 \\
2 \\
2
\end{array}\right), \mathbf{p}_{2}=\left(\begin{array}{l}
2 \\
2 \\
9
\end{array}\right), \mathbf{p}_{3}=\left(\begin{array}{l}
2 \\
5 \\
2
\end{array}\right), \mathbf{p}_{4}=\left(\begin{array}{l}
2 \\
2 \\
2
\end{array}\right) .
\end{aligned}
$$


It can be verified that this set satisfies $C A R P$. However, for $t=1$ or 3 we necessarily obtain $\mathbf{q}_{4} H^{1} \mathbf{q}_{t}$ or $\mathbf{q}_{4} H^{2} \mathbf{q}_{t}$, while $\mathbf{q}_{t} \geq \mathbf{q}_{4}$ and $\mathbf{q}_{t} \neq \mathbf{q}_{4}$. We obtain this last result in two steps:

Step 1: $C A R P$ consistency requires (for $m \neq l$ and $s, t \in\{1,2,3\}$ )

$\mathbf{q}_{1} H_{0}^{m} \mathbf{q}_{2}, \mathbf{q}_{2} H_{0}^{m} \mathbf{q}_{3}$ and $\mathbf{q}_{3} H_{0}^{l} \mathbf{q}_{2}, \mathbf{q}_{2} H_{0}^{l} \mathbf{q}_{1}$, while not $\mathbf{q}_{s} H_{0}^{1} \mathbf{q}_{t}$ and $\mathbf{q}_{s} H_{0}^{2} \mathbf{q}_{t}$.

The reasoning goes as follows. First, because for all $s, t \in\{1,2,3\}$ we have $\mathbf{p}_{s}^{\prime} \mathbf{q}_{s}>\mathbf{p}_{s}^{\prime} \mathbf{q}_{t}$, rule (i) of Definition 6 implies $\mathbf{q}_{s} H_{0}^{1} \mathbf{q}_{t}$ or $\mathbf{q}_{s} H_{0}^{2} \mathbf{q}_{t}$ and rule (iv) excludes $\mathbf{q}_{s} H_{0}^{1} \mathbf{q}_{t}$ and $\mathbf{q}_{s} H_{0}^{2} \mathbf{q}_{t}$. Next, because $\mathbf{p}_{1}^{\prime} \mathbf{q}_{1}>\mathbf{p}_{1}^{\prime}\left(\mathbf{q}_{2}+\mathbf{q}_{3}\right)$, rule (iv) of Definition 6 excludes $\mathbf{q}_{2} H_{0}^{1} \mathbf{q}_{1}$ and $\mathbf{q}_{3} H_{0}^{2} \mathbf{q}_{1}$ and, conversely, $\mathbf{q}_{2}$ $H_{0}^{2} \mathbf{q}_{1}$ and $\mathbf{q}_{3} H_{0}^{1} \mathbf{q}_{1}$. Similarly, $\mathbf{p}_{3}^{\prime} \mathbf{q}_{3}>\mathbf{p}_{3}^{\prime}\left(\mathbf{q}_{1}+\mathbf{q}_{2}\right)$ excludes $\mathbf{q}_{2} H_{0}^{1} \mathbf{q}_{3}$ and $\mathbf{q}_{1} H_{0}^{2} \mathbf{q}_{3}$ and, conversely, $\mathbf{q}_{2} H_{0}^{2} \mathbf{q}_{3}$ and $\mathbf{q}_{1} H_{0}^{1} \mathbf{q}_{3}$. We conclude that $C A R P$ consistency requires (6) and (7).

Step 2: because $\mathbf{p}_{4}^{\prime} \mathbf{q}_{4}>\mathbf{p}_{4}^{\prime} \mathbf{q}_{2}$, rule (i) of Definition 6 implies $\mathbf{q}_{4} H_{0}^{1} \mathbf{q}_{2}$ or $\mathbf{q}_{4}$ $H_{0}^{2} \mathbf{q}_{2}$. Together with (6) and (7), this implies $\mathbf{q}_{4} H^{m} \mathbf{q}_{3}$ (and not $\mathbf{q}_{3} H^{m} \mathbf{q}_{4}$ ) or $\mathbf{q}_{4} H^{l} \mathbf{q}_{1}$ (and not $\mathbf{q}_{1} H^{l} \mathbf{q}_{4}$ ), which gives the result.

We conclude that a data rationalization of the given set requires nonmonotone individual utility functions: for $t=1$ or 3 , we have $W^{1}\left(\mathbf{q}_{4}\right)>$ $W^{1}\left(\mathbf{q}_{t}\right)$ or $W^{2}\left(\mathbf{q}_{4}\right)>W^{2}\left(\mathbf{q}_{t}\right)$, while $\mathbf{q}_{t} \geq \mathbf{q}_{4}$ and $\mathbf{q}_{t} \neq \mathbf{q}_{4}$.

\section{References}

[1] Afriat, S. (1967), "The construction of utility functions from expenditure data", International Economic Review, 8, 67-77.

[2] Brown, D. and R. Matzkin (1996), "Testable restrictions on the equilibrium manifold", Econometrica, 64, 1249-1262.

[3] Brown, D. and C. Shannon (2000), "Uniqueness, Stability and Comparative Statics in Rationalizable Walrasian Markets," Econometrica, 68, 1529-1540.

[4] Browning, M, F. Bourguignon, P.-A. Chiappori and V. Lechene (1994), "Income and Outcomes: A Structural Model of Intrahousehold Allocations", Journal of Political Economy, 102, 1067-1096. 
[5] Browning, M. and P.-A. Chiappori (1998), "Efficient intra-household allocations: a general characterization and empirical tests", Econometrica, $66,1241-1278$.

[6] Bourguignon, F., Browning, M and P.-A. Chiappori (2008), "Efficient intra-household allocations and distribution factors: implications and identification", Review of Economic Studies, forthcoming.

[7] Carvajal, A., I. Ray and S. Snyder (2004), "Equilibrium behavior in markets and games: testable restrictions and identification", Journal of Mathematical Economics, 40, 1-40.

[8] Cherchye, L., B. De Rock, J. Sabbe and F. Vermeulen (2008), "Nonparametric tests of collectively rational consumption behavior: an integer programming procedure", Journal of Econometrics, forthcoming.

[9] Cherchye, L., B. De Rock and F. Vermeulen (2007), "The collective model of household consumption: a nonparametric characterization", Econometrica, 75, 553-574.

[10] Cherchye, L., B. De Rock and F. Vermeulen (2008), "The revealed preference approach to collective consumption behavior: testing, recovery and welfare analysis", IZA Discussion Papers 3062, Institute for the Study of Labor (IZA), version July 2008.

[11] Cherchye, L., B. De Rock and F. Vermeulen (2008), "Analyzing Cost Efficient Production Behavior Under Economies of Scope: A Nonparametric Methodology", Operations Research, 56, 204-221.

[12] Chiappori, P.-A. (1988), "Rational household labor supply", Econometrica, 56, 63-89.

[13] Chiappori, P.-A. (1992), "Collective labor supply and welfare", Journal of Political Economy, 100, 437-467.

[14] Chiappori, P.-A. and I. Ekeland (2006), "The micro economics of group behavior: general characterization", Journal of Economic Theory, 130, $1-26$.

[15] Chiappori, P.-A. and I. Ekeland (2008), "The micro economics of efficient group behavior: identification", Econometrica, forthcoming. 
[16] Diewert, W. E. (1973), "Afriat and Revealed Preference Theory", Review of Economic Studies, 40, 419-426.

[17] Forges, F. and E. Minelli (2006), "Afriat's theorem for general budget sets", CESifo Working Paper Series No. 1703, Center for Economic Studies and Ifo Institute for Economic Research.

[18] Houthakker, H. S. (1950), "Revealed Preference and the Utility Function", Economica, 17, 159-174.

[19] Kannai, Y. (1977), "Concavifiability and constructions of concave utility functions", Journal of Mathematical Economics, 4, 1-56.

[20] Richter, M.K. and K.-C. Wong, "Concave utility on finite sets", Journal of Economic Theory, 115, 341-357.

[21] Samuelson, P. A. (1938), "A Note on the Pure Theory of Consumer Behavior", Economica, 5, 61-71.

[22] Starr, R. (1969), "Quasi-equilibria in markets with non-convex preferences", Econometrica, 37, 25-38.

[23] Starret, D. (1972), "Fundamental nonconvexities in the theory of externalities", Journal of Economic Theory, 4, 180-199.

[24] Mas-Colell, A., M. Whinston and J. Green (1995), Microeconomic Theory, Oxford, Oxford University Press.

[25] Varian, H.R. (1982), "The nonparametric approach to demand analysis", Econometrica, 50, 945-972.

[26] Varian, H.R. (2006), "Revealed preference", in M. Szenberg, L.Ramrattan and A.A. Gottesman (eds.), Samuelsonian economics and the 21st century, Oxford University Press. 